\title{
An Adaptive Virtual Reality System for the Treatment of Adjustment Disorder and Complicated Grief: 1-year Follow-up Efficacy data
}

\author{
Soledad Quero ${ }^{\mathrm{a}, \mathrm{c*}}$, Mar Molés ${ }^{\mathrm{a}}$, Daniel Campos ${ }^{\mathrm{a}}$, Sabrina Andreu-Mateu ${ }^{\mathrm{a}}$, Rosa M. \\ Baños $^{\mathrm{b}, \mathrm{c}}$, Cristina Botella ${ }^{\mathrm{a}, \mathrm{c}}$ \\ ${ }^{a}$ Departamento de Psicología Básica, Clínica y Psicobiología, Universitat Jaume I; Castellón, Spain \\ ${ }^{b}$ Departamento de Personalidad, Evaluación y Tratamientos Psicológicos, Universidad de Valencia, \\ Valencia, Spain \\ ${ }^{\mathrm{c}}$ CIBER de Fisiopatología de la Obesidad y Nutrición (CIBERON), Instituto Salud Carlos III, Madrid, \\ Spain
}

\begin{abstract}
Adjustment disorders (AD) and complicated grief (CG) are serious mental conditions that have a high prevalence and are associated with significant impairments in social and work functioning. Recently, these categories have been better specified in the new ICD-11 proposal. Empirical research on the efficacy of treatments for these problems is scarce. This study aims to offer long-term efficacy data from a between-groups controlled study that compares two treatment conditions (AD-protocol applied in a traditional way: $\mathrm{N}=18$; and the same protocol supported by virtual reality-VR-: $\mathrm{N}=18$ ) and a waiting list (WL) control group $(\mathrm{N}=18)$. Both treatment conditions resulted in statistically significant improvements on both primary and secondary outcome measures, with large effect sizes, and this improvement did not occur in the WL. These changes were maintained in both treatment conditions in the medium (6-month) and long term (12-month follow-up). Larger effect sizes were achieved in the VR condition in the long term. Furthermore, clinically significant change estimations on the primary outcome measures showed an advantage for the VR condition This is the first controlled study to compare a traditional face-to-face protocol for the treatment of stress-related disorders to the same protocol supported by VR and a WL control group.
\end{abstract}

\section{Key Practitioner Message:}

- A CBT protocol has been designed and developed for AD.

- This protocol applied traditionally and with VR was more effective than a WL.

- Changes were maintained in both treatment conditions at 6- and 12-month follow-ups.

- VR seems to be a promising tool to deliver the elaboration/exposure component for AD and CG.

Key words: Adjustment Disorder, Complicated Grief, Prolonged Grief Disorder, CBT, Virtual reality, Positive psychology strategies.

\footnotetext{
* Corresponding author:

Soledad Quero PhD.

Departamento de Psicología Básica, Clínica y Psicobiología. Universitat Jaume I.

Avda. Vicente Sos Baynat s/n 12071 Castellón (Spain)

Phone: +34 $964387641 \quad$ Fax: +34 964729267

E-mail: squero@uji.es
} 


\section{INTRODUCTION}

Adjustment disorders (AD) and complicated grief (CG) are two categories that have traditionally been poorly specified by the DSM and ICD-10. In the last edition (DSM-5, APA, 2013), AD was classified for the first time in the category of "Trauma and stress related disorders", along with another important disorder, posttraumatic stress disorder (PTSD). However, the DSM-5 has been criticized for the lack of specific symptom descriptions and the difficulty of distinguishing between AD and normal adaptive stress reactions (Casey, 2014). In the case of CG, DSM-5 has introduced criteria for persistent complex bereavement disorder also categorized as one of the "other specified trauma- and stressor-related disorder" while the CG, was placed in the Research section (III) as a condition requiring further study.

More recently, the ICD-11 work group proposed that these two diagnostic categories to be included for the first time in the new chapter "Disorders Specifically Associated with Stress" and have provided a clearer definition of symptoms (Maercker et al., 2013). AD is defined as a maladaptive reaction to a negative stressful life event or life change, characterized by symptoms of preoccupation, such as excessive worry, recurrent and distressing thoughts about the stressor, or constant rumination about its implications. There is a failure to adapt, i.e., the symptoms interfere with everyday functioning, such as difficulties concentrating or sleep disturbances resulting in performance problems. These symptoms can also be associated with loss of interest in work, social life, caring for others, leisure activities, resulting in impairments in social or occupational functioning. Prolonged grief disorder is defined as a disturbance in which, following the death of a person close to the bereaved, there is a persistent and pervasive yearning or longing for the deceased, or a persistent preoccupation with the deceased that extends for an abnormally long period beyond the expected social and cultural norms (e.g., at least 6 months, or longer, depending on cultural and contextual factors) and is sufficiently severe to cause significant impairment in the person's functioning. The response can also be characterized by difficulties in accepting the death, feeling that one has lost a part of one's self, anger about the loss, guilt, or difficulty in engaging with social or other activities. In this article, we use the term CG to refer to the Prolonged Grief disorder proposed by the ICD-11. Participants with persistent complex bereavement disorder were not included (Boelen \&Smid, 2017).

However, at the time the present study was conducted, the classifications mentioned above were not available. Therefore, the DSM-IV-TR (APA, 2000) and 
ICD-10 (WHO, 1992) criteria for AD were followed. In the case of CG, according to the ICD-10 classification, we considered it as an AD. Specifically, the ICD-10 allows the codification of bereavement as an $\mathrm{AD}$ when the reaction to the death of a loved one is considered abnormal because of its manifestations or content, or when this reaction is still intense more than 6 months after the death.

$\mathrm{AD}$ is among the diagnoses most widely used by psychiatrists and psychologists worldwide (Maercker et al., 2013). Recently, Yaseen (2017) estimated AD prevalence data in outpatient psychiatric clinics in values around $11 \%$. Regarding CG, a recent systematic review and meta-analysis on the prevalence of prolonged grief disorder in adult bereavement conducted by Lundorff, Holmgren, Farver-Vestergaard and O'Connor. (2017) revealed a pooled prevalence of $9.8 \%$. In addition to the high prevalence of AD (Carta, Balestrieri, Murru \& Hardoy, 2009), this problem is associated with significant impairment in social and work functioning, causing a high percentage of sick leave (Van der Klink, Blonk, Schene \& van Dijk, 2003). Furthermore, $\mathrm{AD}$ is the most common diagnosis in people with self-harm behaviors (Casey, 2009); up to $25 \%$ of adolescents with AD perform suicidal behaviors (Pelkonen, Marttunen, Henriksson \& Loongvist, 2007), with this percentage reaching $60 \%$ in adults (Kryzhananovskaya \& Canterbury, 2001). Results obtained more recently by Casey, Jabbar, O'Leary and Doherty (2015) indicate that AD is a potentially serious condition that can present life-threatening features, with similar percentages of patients with AD and depressive episodes reporting suicidal ideation or behavior. In addition, the occurrence of suicidality at a lower symptom score in AD suggests that this group is more vulnerable. Along the same lines, CG is associated with intense suffering and the risk of developing mental and physical health problems (Stroebe, Schut \& Stroebe, 2007), increasing the risk of hospitalization (Li, Laursen, Precht, Olsen, \& Mortensen, 2005), and being associated with medical comorbidity (Prigerson et al., 1997), suicidality (Latham \& Prigerson, 2004), and greater work and social impairment. It is a debilitating and chronic disorder (Simon et al., 2007).

Therefore, evidence-based psychological treatments for AD and CG are needed. Few intervention studies are available on AD (Casey \& Bailey, 2011). In addition, the few studies conducted using between-group designs have been carried out from different therapeutic approaches: mirror therapy (González-Jaimes \& Turnbull-Plaza, 2003), problem solving and temporal contingency approach (van der Klink et al., 2003), CBT (Sterinhardt \& Dolbier; 2008), brief group psychodynamic therapy (Ben-Itzhak et 
al., 2012), Body-Mind-Spirit psychotherapy (Hsiao et al., 2014), meditation training (Srivastava, Talukdar \& Lahan, 2011);). Although overall they reported a decrease of symptoms, many of them have important methodological limitations. Some use techniques with no empirical support (e.g., Hsiao et al., 2014), whereas others do not describe the samples used sufficiently (e.g., González-Jaimes \& Turnbull-Plaza, 2003), and/or they do not include control groups (Ben-Itzhak et al., 2012) or the ones they use are inadequate (e.g., Srivastava et al., 2011). Finally, other studies focus on a very restrictive objective, such as limiting the duration of sick leaves (van der Klink et al., 2003), or their intervention proposal is limited to counseling or discussion about possible problem-solving strategies, and/or the sample is composed of university students (Sterinhardt \& Dolbier, 2008).

Regarding treatment for CG, Neimeyer has a number of publications about treatments for this problem. His approach (Neimeyer, 2000) focuses on the reconstruction of meaning after loss (Neimeyer \& Currier, 2009). Results from the 11 studies included in the review study by Currier, Holland and Neimeyer (2010) showed that CBT-based interventions were more effective than other commonly practiced therapies. However, after accounting for the influence of researcher allegiance, differences between therapies became smaller and were no longer statistically significant. Compared to no-treatment control groups, CBT-based interventions produced benefits immediately after intervention, but they did not yield statistically significant overall effects at follow-up. Therefore, the authors concluded that there is preliminary evidence for the helpfulness of CBT-based interventions for bereaved persons, but studies on the relative efficacy of different cognitive-behavioral change strategies, as well as other orientations, are still needed. As indicated in more recent review studies (Shear, 2015; Crunk, Kurke \& Robinson III, 2017), several randomized controlled trials have shown that a short-term approach called complicated grief treatment is the treatment that has been most extensively studied so far (e.g., Boelen, Keijser, vand den Hout \& van den Hout, 2007; Bryant, Kenny, Joscelyne, et al., 2014; Shear, Frank, Houck \& Reynolds, 2005; Shear et al., 2014). The treatment includes two main objectives: restoring effective functioning by generating enthusiasm and creating plans for the future and helping patients find a way to think about the death without having intense feelings of anger, guilt or anxiety (Shear, 2015).

Based on the aforementioned, we can conclude that no specific protocol exists for $\mathrm{AD}$ because none of the experimental studies reviewed above propose a treatment 
specifically designed for this problem. Therefore, we developed an AD-protocol to treat AD and CG (Botella, Baños \& Guillén, 2008) that includes CBT techniques and positive psychology strategies. Furthermore, it is supported by virtual reality (VR). Several systematic reviews (Freeman et al., 2017; Gregg \& Tarrier, 2007; McCann et al., 2014; Meyerbroker \& Emmelkamp, 2010) and a number of meta-analytic studies (Opriş et al., 2011; Parsons \& Rizzo, 2008; Powers \& Emmelkamp, 2008) concluded that the most well-established finding is that VR exposure-based treatments can reduce anxiety disorders and PTSD. In the case of Opris et al. (2011) and Morina et al. (2015), the authors also found that VR has a powerful real-life impact, similar to that of the classical evidence-based treatments, and good stability in the results over time. Additionally, meta-analyses and systematic reviews specifically addressed to stressrelated disorders (mainly PSD) (Botella, Serrano, Baños \& Garcia-Palacios, 2015; Gonçalves, Pedrozo, Coutinho, Figueira \& Ventura, 2012; Motraghi, Seim, Meyer \& Morissete, 2014), have also shown the usefulness of VR environments for applying the exposure technique in treating these disorders. Finally, other exposure-based approaches, such as EMDR, have also shown their efficacy in the treatment of distressful experiences that failed to meet the criteria for PTSD (Cvetek (2008).

The present study uses the EMMA's World VR system (Botella et al., 2006) to apply elaboration/exposure technique. This system was developed as part of a European project (Engaging Media for Mental Health Applications, IST-200-39192). EMMA's World is an open and flexible adaptive VR device that adjusts its contents to the patient's needs. This flexibility makes it possible to use this VR system to treat CG and a variety of $\mathrm{AD}$ cases, regardless of the stressful event that triggered them (e.g., a divorce, a firing, a serious disease, economic problems, etc.). Preliminary efficacy data for this protocol already exist in two case studies (Andreu-Mateu, Botella, Quero, Guillén \& Baños, 2012; Botella, Osma, García-Palacios, Guillén \& Baños, 2008), and in a between-group study (Baños et al., 2011) that included participants with different stress-related disorders (PTSD, CG and AD). The results showed that traditional CBT programs were equally as effective as a CBT program supported by EMMA's World. However, no control group was included in this study, and no follow-up data were available. The aim of the present study is to offer long-term efficacy data from two ADtreatment protocols (an AD-treatment protocol supported by VR -EMMA's World-and an $\mathrm{AD}$-protocol applied in a traditional - face-to face way) compared to a waiting list control group in an RCT. A secondary aim is to explore and compare both ways of 
delivering the AD-protocol. First, it is hypothesized that both treatment conditions will significantly reduce primary symptoms of $\mathrm{AD}$ and $\mathrm{CG}$, compared to the waiting list control group. Second, both treatment conditions will show efficacy and no statistically significant differences will be found between them. Finally, the therapeutic gains obtained in both conditions will be maintained at 1-year follow-up.

\section{METHOD}

\subsection{Design}

A three-armed single-blind RCT was conducted. Participants were randomized into three groups: 1) EMMA condition, 2) Traditional condition, and 3) Waiting list (WL) control condition. Participants in the control group were randomly assigned to one of the two treatment conditions after spending time on the waiting list (6 weeks) for ethical reasons. This study was conducted following the CONSORT statement (Consolidated Standards of Reporting Trials, http://www.consort-statement.org) and received approval from the Ethics Committee of Universitat Jaume I (Castellón, Spain) Participants were assessed at pre-treatment, post-treatment, and 6- and 12-month follow-ups. Figure 1 shows the flowchart for the study.

\subsection{Participants}

This study was conducted at the Emotional Disorders Clinic at Universitat Jaume I in Castellón and Valencia (Spain). Participant recruitment was carried out through advertisements (mail, posters, radio, and press) about the study developed in our clinic. Other participants came to seek help at our clinic, and others were referred from public mental health services.

Inclusion criteria were: age between 18 and 65 years old; meeting $\mathrm{AD}$ criteria according to the DSM-IV-TR (APA, 2000) and ICD-10 (WHO, 1992) or Prigerson and Jacobs' (2001) criteria for CG. Exclusion criteria were: current alcohol or drug dependence or abuse; psychosis or a severe personality disorder; a severe organic illness; malingering, showing obvious "secondary gains" (e.g., financial compensation, avoidance of work, etc.); and currently being treated in a similar treatment program. Receiving pharmacological treatment was not an exclusion criterion, but any increase and/or change in the medication during the study period implied the participant's exclusion from subsequent analyses. A decrease in pharmacological treatment was accepted. In the present study, as we have mentioned before, following ICD-10 criteria, cases of CG were considered an AD subtype, and patients with both diagnoses were included. Additionally, Prigerson and Jacobs' (2001) criteria were considered in the CG 
cases. Table 1 shows participants' sociodemographic and clinical data for each group and for the total sample. The type of stressful event experienced by the AD patients was: break-up relationship or divorce $(n=14 ; 25.93 \%)$, own or family health problems $(\mathrm{n}=11 ; 20.37 \%)$, work/study problems $(\mathrm{n}=11 ; 20.37 \%)$, family problems $(\mathrm{n}=12$; $21.82 \%)$, or grief $(n=6 ; 10.91 \%)$.

INSERT FIGURE 1 ABOUT HERE

\section{INSERT TABLE 1 ABOUT HERE}

\subsection{Measures}

\subsubsection{Diagnostic}

Diagnostic Interview Adjustment Disorder (Andreu-Mateu, Botella, Baños \& Quero, 2008). This semi-structured interview was developed by our research team for the assessment of $\mathrm{AD}$, based on data available in the literature, the diagnostic criteria from both the ICD-10 and the DSM-IV-TR, and the SCID-IV interview (First, Spitzer, Gibbon and Williams 1999). The presence and severity of 28 symptoms related to AD are assessed on a scale from 0 to $8(0=$ Nothing at all; $8=$ Very severe $)$. This interview is currently undergoing a validation process. An optional section for evaluating Prigerson and Jacobs' (2001) criteria for complicated grief is also included. In the present study, the number of symptoms was analyzed as an outcome measure.

\subsubsection{Primary outcome measures}

Inventory of Complicated Grief (ICG) (Prigerson et al. 1995). This instrument assesses the symptoms that characterize complicated grief, and it differentiates individuals who suffer from complicated grief symptoms, cannot adapt adequately to the death of a loved one, and present important functional alterations in their daily lives from those who are following a normal bereavement process. It consists of 19 items scored on a 5-point Likert scale ranging from 0 ("Never") to 4 ("Always"). Scores above 25 indicate a significantly higher degree of interference in general, mental, and social functioning, worse physical health, and higher levels of physical pain. Prigerson et al. (1995) obtained high internal consistency, test-retest reliability, and convergent and criterion validity.

Adaptation of the ICG, Inventory of Stress and Loss (ISL) (Quero, Mor, Molés, Rachyla, Baños \& Botella, in preparation), in order to measure stress and loss symptoms derived from stressful situations. This instrument has been adapted by our 
team to better assess the stressful situations that negatively interfere with people's lives. The adaptation was based on the assumption that a stressful life event always entails some kind of loss for the person. The instrument was made up of the same items included in the ICG, but the words referring to the "dead person" were substituted by words referring to the "person/situation" that had been lost due to a stressful event (e.g., loss of a job, divorce, loss of health, etc.). Two items were removed because they were not pertinent to assessing $\mathrm{AD}$; therefore, the final questionnaire included 17 items. Preliminary validation data (Quero, Molés, Mor, Baños \& Botella, 2014) showed excellent Cronbach coefficients in both general (0.91) and clinical AD (0.86) Spanish populations. Test-retest reliability was also excellent (0.90).

Beck Depression Inventory (BDI-II) (Beck Depression Inventory, Beck, Steer \& Brown 1996; Spanish adaptation by Sanz, Navarro \& Vázquez, 2003). This is one of the most widely used instruments for assessing depression symptoms. It includes 21 items, each with four possible answers (0-3). The participants were asked to choose which statements best described their mood states in the past 2 weeks. This instrument assesses mainly cognitive aspects of depression, along with behavioral and physiological symptoms. It establishes 4 quantitative ranges of depression: from 0 to 9 (absent or minimal), from 10 to 18 (from light to moderate), from 19 to 29 (from moderate to severe), and from 30 to 63 (severe). It has high internal consistency ( $\alpha=.87$ in the general population and $\alpha=.89$ for the clinical population) (Sanz, García-Vera, Espinosa, Fortún, \& Vázquez, 2005; Sanz, Perdigón \& Vázquez, 2003).

\subsubsection{Secondary Outcome Measures}

Posttraumatic Growth Inventory (PTGI) (Tedeschi \& Calhoun, 1996). This inventory is composed of 21 items that assess the positive changes that can be experienced by people after suffering a traumatic or adverse event, using a 0-5 scale (0 $=$ "I did not experience this change at all"; $5=$ "I experienced this change to a great extent"). It includes 5 scales indicating different growth dimensions: related to others (7 items), new possibilities (5 items), personal strengths (4 items), spiritual changes (2 items), and life appreciation ( 3 items). Furthermore, a global score can be calculated by adding together the scores obtained on all the responses. High internal consistency levels for the 5 scales and for the total score have been found (Tedeschi \& Calhoun 1996; So-kum Tang, 2007, Ho, Wing Chu \& Yiu 2008). High scores on this inventory 
indicate a higher degree of perceived posttraumatic growth. Preliminary data validation of the PTGI adaptation in a Spanish clinical sample with AD and CG (Molés, Quero, Nebot, Rachyla \& López, 2014) showed high internal consistency (0.92) and test-retest reliability (0.95).

Maladjustment Scale (MS) (Adapted from Echeburúa, Corral \& FernándezMontalvo, 2000). This instrument assesses the level of impairment that the problem and its consequences are causing in the patient's different life areas (work, social life, leisure, partner, family, and global impairment), using 0-8 scales where 0 indicates "Not impaired" and 8 indicates "severely impaired". This scale offers good psychometric properties, and it is sensitive to the effects of treatment. In the present study, only global impairment is presented

Clinician Severity Scale (Adapted from ADIS-IV Interview by Di Nardo, Brown \& Barlow, 1994). The therapist makes a global evaluation of the patient's interference and severity on a scale from 0 ("Not impaired or without symptoms") to 8 ("Severely impaired or Very severe symptoms").

\subsection{Treatment}

The AD-protocol developed by Botella, Baños and Guillén (2008) was composed of 6 weekly therapy sessions lasting approximately 1.5 hours each. Two additional sessions were optional, depending on the participant's therapeutic needs. Although AD and CG are separate disorders in the ICD-11, they are included in the same category of disorders associated with stress. They have common characteristics, and so they share similar therapeutic objectives. In this regard, the main aim of the treatment applied in this study for both conditions was the reconstruction of meaning after loss, in the case of CG, and of the stressful event (e.g., break-up relationship, health problems), in the case of $\mathrm{AD}$ patients, in order to achieve a more positive meaning, thus allowing post-traumatic growth and learning from the negative experience. The AD-protocol includes the following therapeutic components.

\section{1) Psychoeducation}

The patient is provided with information about common reactions to a stressful event and a theoretical model that explains how the problems occur and how they are maintained. Throughout the treatment sessions, the psychoeducation component is aimed at reformulating the patient's interpretation of difficulties and his/her attitude toward problems, teaching more positive ways to manage stressful situations and introducing an alternative model explaining the onset and maintenance of AD. 


\section{2) Exposure, elaboration/processing of the stressful event}

The main objective of this component is to get the person to elaborate on, find alternative meanings for, and learn from the stressful experience. This component was applied in two ways: 1) Using VR (EMMA's World). Patients' narratives of the experience are conducted within EMMA's World (described below), which helps participants to evoke, confront, and process the negative emotions associated with the negative event, while remaining in a safe virtual space. 2) Traditional format. Patients' narratives of the experience are conducted within a traditional face to face therapy context.

In applying this component, instructions were given to the patient about the importance of being aware of emotions, thoughts, and behaviors without judging them. The final aim was for the participant to change the negative meaning of the stressful event into a more positive one while learning about the negative experience. In vivo exposure was also used to confront avoided situations or people related to the stressful event.

\section{3) Strategies from Positive Psychology}

Several strategies from Positive Psychology were included in the protocol in order to promote elaboration on, assimilation of, and learning from the experienced event. These strategies were the following: 1) "Problem acceptance training" (Popper, 1995). Lessons about problems as inherent to the human condition were presented to the patients. Elements include the usefulness of having problems, the significance of problem-solving, and the importance of accepting problems as endemic to the life experience. 2) “My best virtues or strengths" exercise (Peterson \& Seligman, 2004). In this exercise, it is suggested to the patient that virtues give people the strength and capacity to cope with life. Examples of virtues are offered to the patient, including curiosity, optimism towards the future, sense of humor, etc. Patients have to analyze which ones they think they have and which ones they think it would be useful to develop and promote in order to cope with their lives. 3) "Heuristics" exercise. From a list of heuristics (proverbs, statements, life guidelines), patients choose the ones they find helpful in maintaining and promoting the changes achieved in their lives.

\section{4) Strategies from Neimeyer (2000)}

Several strategies based on Neimeyer's (2000) suggestions for CG were adapted and included in the protocol with the aim of promoting the elaboration/processing of the stressful event: 1) The Book of Life (adapted from Neimeyer, 2000). The aim of the 
book is to help the patient to remember and elaborate on meaningful events that have happened with regard to the adverse event. In writing this book, patients can use not only words, but also symbols (e.g., pictures, drawings, phrases, etc.). Through this process, patients can reflect on the negative situation, the deceased person, the broken relationship, etc. The Book of Life is where the patient can reexamine and sort through the jumble of memories, thoughts, and emotions that often produce confusion, pain and suffering. This process gives the person the opportunity to correct the information that was not well stored in his/her memory; that is, the person has the opportunity to rework the memories in order to create alternative meanings; 2) the "Vital trace" exercise. Through this exercise patients try to deepen their search for positive aspects of the stressful event. According to Neimeyer (2000), people consist of the vestiges of their life experiences, both positive and negative, and all of our experiences offer opportunities to improve ourselves; 3) Projection letter to the future”. This task requires patients to write a letter to themselves from the future. To accomplish this, they first spend 10 minutes a day for 2 to 3 days, imagining what kind of person they will be in 10 years (what they will be doing, where they will live and with whom, what their careers will be, etc.), and then they write the letter to themselves in second person (as if they were writing to a loved one) from the future. Later analysis of the letter will focus on sections that reveal the patient's ability to see "beyond" the current negative situation.

\section{5) Relapse prevention}

The main objective of this component is to maintain and promote therapeutic changes in the future. The therapist reviews the therapeutic objectives achieved by the patient so far, makes a summary of the main therapeutic components worked on throughout the treatment, and resolves doubts and identifies pending issues to work on in the future.

\subsection{Description of EMMA's World}

EMMA's World (Baños et al., 2009; Botella et al., 2006) was developed within a European Union project (Engaging Media for Mental Health Applications, IST-20039192). It consists of an adaptive VR open system that, because of its flexibility, can be used to treat different psychological problems. In the specific case of $\mathrm{AD}, E M M A$ 's World is used to activate and process the emotions and cognitions related to the stressful 
event and expose the patients to aspects they have been avoiding, in order to facilitate the emotional processing of all the aspects related to the stressful event. The underlying logic follows Foa and Kozak’s approach (1986) stating that feared pathological structures need to be activated as much as possible in order to fully process the event. In EMMA's World, the experienced stressful event can be "physically" represented to the patient using different symbols, such as images, 3-D objects, sounds, music, etc., that best reflect the emotions and thoughts related to the experience. Furthermore, personal elements that are significant to the patient can be introduced in the system (e.g., pictures). An important element is the Book of Life, a virtual book in which the patient can compile all of the elements that help him/her to represent the most relevant moments, people or situations related to the negative experience. EMMA's world also includes five different pre-defined scenarios or "landscapes": a desert, an island, a threatening forest, a snow-covered town, and meadows. These environments were designed to stimulate different emotions (relaxation, elation, sadness, etc.). Their specific use depends on the context of the session and can be selected by the therapist in real time. The therapist can also modify various aspects of these landscapes in real time (e.g., the time of day, whether it is raining or foggy, etc.) with the aim of reflecting and enhancing the emotion the user is experiencing, or inducing certain emotions. In sum, the different elements included in the system and the landscapes of EMMA's World are designed to help patients confront, accept, and manage the previous emotions and negative experiences in their lives, and find and develop new meanings and positive emotions, while working in a timeless place and a safe therapeutic context. More detailed descriptions of EMMA's World can be found elsewhere (Baños et al., 2009; Baños et al., 2011; Botella et al., 2006; Botella, Quero et al., 2006).

\subsection{Technical aspects}

The following devices were used: two PCs, a large screen on which the environment was projected, two projectors, a wireless pad, and a system of speakers. These devices were placed in a $5 \times 9$ meter room. PC\#1 had the graphical output from its graphic card connected to two projectors (with a resolution of $1024 \times 768$ pixels and a power of 2000 lumens). They were used to project the environment on a horizontal methacrylate screen measuring $4 \times 1.5$ meters, placed in the middle of the room. The patient could see the virtual environment, interact with it, and navigate through it using the wireless pad. The therapist could control the application and the features of the 
virtual environment that were shown to the patient with PC\#2. The sound system consisted of several speakers distributed in the room to conform to a 5.1 configuration.

\subsection{Procedure}

All the participants who met the inclusion criteria signed the informed consent to participate in the study. Then they underwent two assessment sessions lasting 1.5 hours each. In the first assessment session, the diagnosis of AD or CG was confirmed, and self-report measures were completed. In the second assessment session, the therapeutic goals were established. The randomization of the participants took place after the assessment sessions were conducted. Therapists and participants involved in the trial were blind to treatment allocation during the assessment. Participants were assigned to either the EMMA $(\mathrm{N}=18)$, Traditional $(\mathrm{N}=18)$, or Waiting list control $(\mathrm{N}=18)$ conditions based on a computer generated randomization list created by the "Random Allocation Software"; version 1.0. Then participants assigned to the treatment conditions were treated in 6 to 8 treatment sessions lasting approximately 1.5 hours each, with the only difference being the use of VR for the processing/elaboration of the stressful event in the EMMA condition. Participants included in the control condition did not receive any intervention during a 6-week period, after which they were assessed again and then offered the possibility of receiving one of the two treatment conditions. Once the treatment was over, all the participants were assessed again at post-treatment and at 6 and 12-month follow-ups.

Five therapists participated in the study. They had a PhD or a Master's degree in Psychology. All of them were trained in VR techniques and CBT programs for Emotional Disorders. In addition, they had received training in this treatment protocol by senior clinicians. They were supervised by senior clinicians with $\mathrm{PhDs}$ in weekly sessions. Moreover, all of the assessment and treatment sessions were video-taped in order to supervise the performance of each therapist.

\subsection{Data Analyses}

Group differences at pre-treatment on demographic data and clinical variables were evaluated using chi-square tests for categorical variables and one-factor analyses of variance (ANOVAs) for continuous data. Intent-to-treat (ITT) mixed models analyses without any ad hoc imputation were used to account for missing data (Chakraborty \& $\mathrm{Gu}$, 2009). A mixed model approach is appropriate for RCTs with multiple time points and pre-to post-only designs, it does not assume that the last measurement is stable, and it is conducted using all available observations (Gueorguieva, \& Krystal, 2004; Salim, 
Mackinnon, Christensen, \& Griffiths, 2008). A linear mixed model for each outcome measure was implemented with time (pre and post) as within-group factor and group (EMMA, Traditional and WL) as between-group factor using the MIXED procedure with a random intercept for subject. Because the random effect had one level, an identity covariance structure was specified to model the covariance structure of the intercept. Separate mixed-model analyses were performed to examine changes from baseline in each intervention at posttest and 6- and 12-month follow-ups. Significant effects were followed up with pairwise contrasts. Effect sizes (Cohen's $d$ ) were calculated for within- and between-group comparisons, based on Botella and SánchezMeca (2015). Finally, Chi-squared tests were performed based on completers in order to examine the differential clinically significant improvement rates, following the Jacobson and Truax (1991) indexes, for the primary outcomes scores at post-treatment and 6- and 12-month follow-ups in the two treatment conditions just considering those participants who completed the treatment protocol. All statistical analyses were performed using the IBM SPSS version 23 for Windows.

\section{RESULTS}

\subsection{Participant flow and attrition}

Participants were recruited between January 2009 and April 2011. Initially, as the flow diagram shows (see Figure 1), 97 people were interested in the study, and 67 of them were assessed for eligibility criteria. Thirteen participants were excluded from the study for several reasons (see Figure 1). Thus, 54 participants were randomly allocated to each experimental condition (EMMA condition, $\mathrm{N}=18$; Traditional condition, $\mathrm{N}=$ 18; and WL, $\mathrm{N}=18$ ). However, during treatment, 8 participants dropped out of the study. No significant differences in attrition rates were found at post-treatment between conditions. After treatment completion, 1 participant in the traditional condition worsened and was excluded from the study. This participant was successfully treated years ago for an obsessive-compulsive disorder that reappeared in the follow-up assessment period of the present study due to a new stressful situation in his life, and so more therapy sessions were needed. Therefore, in this condition, 14 participants completed the 6-month follow-up assessment. Finally, at 12-month follow-up, a total of 24 participants attended the assessment session: 10 participants in the traditional condition and 14 in the EMMA condition. Data were missing completely at random $(\mathrm{MCAR})(\mathrm{p}>.50)$. 


\subsection{Baseline data and participants characteristics}

Baseline analyses showed no significant differences between the three groups at pre-treatment on any demographic variables, diagnostic subtype, length of the problem, medication intake variables, and outcomes measures, except for the PTGI $(\mathrm{F}(2,51)$ $=5.187, \mathrm{p}<.05)$, where the WL control group scored significantly higher than the two treatment conditions (EMMA and Traditional). No variations in the medication were produced throughout the study.

\subsection{Differential effectiveness of the three experimental conditions: changes in outcome measures at pre and post-treatment}

Primary outcomes

A significant time x group interaction was found for $\operatorname{BDI-II}(F(2,46.78)=3.575$, $p<.05)$ and $I S L / I C G(F(2,44.36)=27.898, p<.05)$. Within-group comparisons showed significant reductions on both primary outcome measures for the Traditional [BDI: $F(1,47.14)=22.650, p<.001$; ISL/ICG: $F(1,44.21)=18.385, p<.001]$ and EMMA [BDI: $F(1,47.14)=17.304, p<.001$; ISL/ICG: $F(1,44.21)=18.129, p<.001$ ], conditions, corresponding to large effect sizes (from 1.02 to 1.24), but no significant change was found in the WL group (see Table 2). For the BDI-II, between-group comparisons revealed that post-treatment scores were significantly lower in the Traditional and EMMA groups, compared to WL, with moderate-large effect sizes (-.89 and -.78, respectively) (see Table 3). For the $I S L / I C G$, between-group comparisons revealed that participants in the Traditional condition scored significantly lower at posttreatment than the WL, with a large effect size $(d=-.92)$ (see table 3 for details).

Secondary outcomes

Regarding PTGI, pre-treatment scores on this measure were entered as a covariate to account for baseline differences. Results indicated a significant time $\mathrm{x}$ group interaction effect $(F(2,44.862)=13.733, p<.001)$. Within-group comparisons revealed a significant pre-to-post change in the two treatment groups $(F(1,45.112)=$ 49.962, $p<.001$, for both groups), with large effect sizes (from -1.62 to -2.05), and no significant change in the WL (table 2). At post-treatment, no statistically significant between-group differences were found (Table 3).

For both the Maladjustment Scale and Clinician Severity Scale, a significant time $\mathrm{x}$ group interaction was found $[(F(2,48.32)=5.911, p<.01)(F(2,25.617)=$ 24.088, $p<.001)$, respectively]. Within-group comparisons revealed significant 
reductions for both treatments [(Maladjustment Scale, Traditional: $F(1,49.19)=33.981$, $p<.001$; EMMA: $F(1,47.89)=21.426, p<.001)($ Clinician Severity Scale, Traditional: $F(1,26.51)=29.997, p<.001$; EMMA: $F(1,24.220)=93.938, p<.001)]$, corresponding to large effect sizes, but no significant change was found in WL (see Table 2). Between-group comparisons showed that post-treatment scores on these measures were significantly lower in both treatments compared to WL, achieving large effect sizes (from -.81 to -1.94) (Table 3).

\subsection{Differential effectiveness of the two treatment conditions: changes in outcome measures at pre-, 6-, and 12-month follow-ups}

Separate mixed model analysis for each primary and secondary outcome measure yielded no significant time $x$ group interaction effect. Within-group comparisons revealed a significant change (from pre- to 6- and 12-month follow-ups, respectively) in both treatments for all primary and secondary outcome measures (all $\mathrm{ps}$ $<.001$ ), corresponding to large effect sizes (see table 2 for details). No statistically significant differences between the two treatments (EMMA vs. Traditional) were found at the 6-month or 12-month follow-up (Table 3). However, larger effect sizes were obtained in the EMMA condition at the 12-month follow-up, compared to the Traditional condition (see Table 2).

\section{INSERT TABLES 2 AND 3 ABOUT HERE}

\subsection{Clinically meaningful improvement}

Table 4 shows the percentages of participants in each condition who were recovered, improved, had no change, or were impaired, according to the ISL/ICG and BDI measures, and based on the classification proposed by Iraurgi (2010) and Kupfer (1991). In general, better results were obtained for the primary outcome measure most closely related to the problem, the ISL/ICG. At post-treatment, more participants (who had completed the treatment) in both conditions were included in the categories recovered or improved. Then, the percentage of recovered improved slightly at the 6month follow-up in both conditions, but in the long term, more participants in the EMMA condition were included in the recovered category, whereas a decrease was found in the Traditional condition. In the case of the BDI-II, again significantly more 
participants were included in the recovered category in the EMMA condition than in the Traditional condition.

\section{INSERT TABLE 4 ABOUT HERE}

\section{DISCUSSION}

The main objective of the present study was to test, in a controlled study, the long-term efficacy of a CBT protocol for $\mathrm{AD}$ and $\mathrm{CG}$ in a traditional format versus a format supported by VR (EMMA's World), and compared to a WL control group. First, data showed that both treatment conditions resulted in statistically significant improvements with large effects sizes on both primary and secondary outcome measures, and this improvement did not occur in the WL control condition. Therefore, the first hypothesis was confirmed. These data agree with those obtained in previous studies (Andreu-Mateu et al., 2012; Baños et al., 2011), and they confirm the usefulness of this psychological treatment protocol supported by VR for AD and CG. These results are also consistent with the conclusions reported by review studies on the efficacy of VR exposure therapy for the treatment of anxiety disorders: systematic reviews (Gregg \& Tarrier, 2007; McCann et al., 2014; Meyerbroker \& Emmelkamp, 2010) and metaanalyses (Morina et al., 2015; Opriş et al., 2012; Parsons \& Rizzo, 2008; Powers \& Emmelkamp, 2008), and by meta-analytic studies specifically addressing the use of VR for stress-related disorders (Botella, Serrano, Baños \& Garcia-Palacios, 2015; Gonçalves, Pedrozo, Coutinho, Figueira \& Ventura, 2012; Motraghi, Seim, Meyer \& Morissete, 2014). Finally, our results are also consistent with those obtained in studies on the traditional treatment for AD (Andrews, 1995; Araoz \& Carrese, 1996; Benton \& Lynch, 2005, Strain, 1995; van der Klink, et al., 2003) and CG (Neimeyer, 2000).

Second, as expected, no statistically significant differences were found between the two treatment groups at any assessment moment. However, at the 12-month followup, additional improvements were observed in the EMMA condition, with larger effect sizes in the long term, compared to the Traditional condition (see Table 2). Similarly, results for clinically significant change estimations indicated an advantage for the EMMA condition on the primary outcome measures (ISL/ICG and BDI), with the percentage of recovered participants being significantly higher at 12-month follow-up in this condition, compared to the traditional one. In any case, it is important to point out that scores on the BDI-II were not very high at pre-treatment. Therefore, the second hypothesis was partially confirmed. 
Finally, as hypothesized, significant improvements achieved in both treatments for all primary and secondary outcome measures were maintained in the medium (6month) and long term (12-month).

Based on the results obtained in the present study, we can conclude that both treatment conditions were effective at post-treatment, but the EMMA condition was slightly superior on the outcome measures and the clinically meaningful change in the long term. Furthermore, results obtained from 18 patients included in this study ( 7 from the traditional condition and 11 from the EMMA condition) after treatment, showed that $66.7 \%$ of them preferred the VR condition, whereas only $33.6 \%$ preferred the traditional one. Our data indicate a preference for the EMMA system, but this result cannot be generalized to all patients since the sample size was small. Moreover, it should be kept in mind that these patients were willing to receive psychological treatment supported by VR, and not all patients would necessarily have a positive opinion about technologies. On the other hand, in the case of these problems, the positive results obtained in the long term are especially relevant, considering the few studies available in the literature about the maintenance of therapeutic gains. Results from this study offer additional support for the positive and lasting effects of VR in the psychological treatment field (e.g., Botella et al., 2007; Emmelkamp et al., 2002; Rothbaum, Hodges, Anderson, Price \& Smith, 2002; Ready, Pollack, Rothbaum \& Alarcón, 2006). Other advantages of the VR environment used in the present study should be pointed out. First, as mentioned in the introduction, EMMA's World adapts its contents in relation to the affective manifestations the users express at any time during the therapy session, resulting in the creation of a personalized and clinically meaningful environment, which, in turn, favors the complete immersion in the narrative and the elaboration and assimilation of the events. Second, the system uses symbols, landscapes and other personalized elements to create a context that "represents or symbolizes" the situation. The use of VR and symbols to conduct exposure/elaboration provides a safe context where patients can experience and confront their problems without the inconvenience of real life. In fact, patients perceive VR exposure to be less aversive than the traditional exposure technique (Baños et al., 2009; Botella et al., 2016).

In summary, this study follows along the lines of existing literature supporting the use of technology (in this case VR) in the field of psychological treatments (Freeman et al. 2017) because, as the data point out, information and communication technologies can improve psychological treatments by providing a cost-effective way to 
deliver empirically validated treatments for a variety of psychological problems (Newman et al., 2011), in this case AD. Undoubtedly, this work contributes to achieving the crucial revolution anticipated through the use of VR in the mental health field (Freeman et al., 2017; Linder et al., 2017). Indeed, studies like the present one indicate that this revolution is already underway.

However, the present study has some limitations that should be highlighted. First, the sample size was small and most of the participants had university degree, limiting the generalization of the results. The level of education seems to be well above average, which might also limit the generalizability of the results. On the other hand, as mentioned before, for the diagnosis DSM-IV-TR and ICD-10 criteria were followed in the present study, and the diagnostic interview for $\mathrm{AD}$ is currently under validation process. The Diagnostic Interview for Adjustment Disorder (DIAD) (Comelius et al., 2014) could not be used because it was not available at the time the study was conducted. In addition, it would have been desirable to include an anxiety measure as a primary outcome. Finally, all participants received the complete AD-protocol, and so is not possible to determine the specific contribution of each therapeutic component to the patients' improvement. Related to this, future studies should consider evaluating whether $\mathrm{AD}$ and $\mathrm{CG}$ patients differ in their response to this treatment protocol, in order to detect the need to tailor the treatment to each stress-related disorder included in the present study.

A future guideline in the field of using technology to improve psychological interventions in $\mathrm{AD}$ and $\mathrm{CG}$ is the use of the Internet to complement the therapy. Preliminary data are already available on the usefulness of an online emotion regulation system (TEO) to apply homework assignments over the Internet in treating AD (Quero, Molés, Pérez-Ara, Botella \& Baños, 2012). This system allows patients to practice the elaboration/processing of the stressful event at home during the period between sessions. A system such as TEO offers great adaptability and flexibility in adjusting the therapeutic homework to each patient's characteristics and needs, focusing on the specific significance of his/her problem.

To conclude, the results obtained in the present study have contributed to increasing the knowledge and available resources to deal with $\mathrm{AD}$ and $\mathrm{CG}$. It is the first controlled study to compare an AD-protocol applied face-to-face to the same protocol supported by VR and a waiting list control group in the treatment of these stress-related disorders. The possibility of having an effective treatment protocol is particularly 
relevant in the $\mathrm{AD}$ field, considering the few controlled studies found in the literature about AD treatment, in addition to its high prevalence (Carta et al., 2009), the impairment in social and work functioning associated with this problem (Van der Klink et al., 2003), the potential AD has of presenting life-threatening features (Casey et al., 2015), and in the long term, the burden for the individual, his/her work place, society, and the economy (Lagerveld et al., 2012).

\section{Acknowledgments}

The research presented in this paper was funded in part by Plan 2011 de Promoción de la Investigación UJI-Proyectos BANCAIXA (P11B2011-20), Ministerio de Economía y Competitividad (Spain) (Plan Nacional I+D+I. PSI2013-41783-R), and CIBER. CIBER Fisiopatología de la Obesidad y Nutrición is an initiative of ISCIII.

\section{References}

American Psychiatric Association (2000). Diagnostic and statistical manual for mental disorders ( $4^{\text {th }}$ edition, revised text) (DSM-IV-TR). Washington: DC.

Andreu-Mateu, S., Botella, C., Quero, S., Guillén, V., \& Baños, R. M. (2012). El tratamiento de los trastornos adaptativos: cuando el estímulo estresante sigue presente. Behavioral Psychology/Psicología Conductual, 20, 323-348.

Andrews, A. (1985). Treatment outlines for the management of anxiety status: The quality assurance project. Australian and New Zealand Journal of Psychiatry, 19, 138-151.

Araoz, D. L., \& Carrese, M. A. (1996). Solution-oriented brief therapy for adjustment disorders: A guide for providers under managed care. New York, N.Y: Brunner/Mazel.

Baños, R. M., Guillén, V., Quero, S., García-Palacios, A., \& Botella, C. (2011). A virtual reality system for the treatment of stress-related disorders: A preliminary analysis of efficacy compared to a standard cognitive. International Journal of Human-Computer Studies, 69, 602-613.

Baños, R. M., Botella, C., Guillén, V., García-Palacios, A., Quero, S., Bretón-López, J., \& Alcañiz, M. (2009). An adaptive display to treat stress-related disorders: The EMMA's world. British Journal of Guidance and Counseling, 37, 347-356.

Beck, A. T., Steer, R. A., \& Brown, G. K. (1996). Manual for the Beck Depression Inventory-II. TX Psychological Corp, San Antonio.

Ben-Itzhak, S., Bluvstein, I., Schreiber, S., Aharonov-Zaig, I., Maor, M., Lipnik, R., \& Bloch, M. (2012). The effectiveness of brief versus intermediate duration psychodynamic 
psychotherapy in the treatment of adjustment disorder. Journal of Contemporary Psychotherapy, 42, 249-256.

Benton, T. D., \& Lynch, J., 2005. EMedicine. Adjustment disorders. http://www.emedicine.com/Med/topic3348.htm. (accessed 07.06.10).

Boelen, P.A., de Keijser, J., van den Hout, M. \& van den Bout, J. (2007). Treatment of complicated grief: A comparison between cognitive-behavioral therapy and supportive counseling. Journal of Clinical and Consulting Psychology, 75, 277-284.

Boelen, P. A., \& Smid, G. E. (2017). Disturbed grief: Prolonged grief disorder and persistent complex bereavement disorder. The BMJ, 357, j2016

Botella, C., Baños, R. M., \& Guillén, V. (2008). Creciendo en la adversidad. Una propuesta de tratamiento para los trastornos adaptativos. In C. Vázquez \& G. Hervás (Eds.), Psicología Positiva Aplicada (pp. 129-154). Bilbao: DDB.

Botella, C., Baños, R. M., Rey, B., Alcañiz, M., Guillén, V., Quero, S., \& García-Palacios, A. (2006). Using an Adaptive Display for the Treatment of Emotional Disorders: A preliminary analysis of effectiveness. Work-In-Progress, CHI. Montréal, Québec, Canada. pp. 586-591.

Botella, C., García-Palacios, A, Villa, H, Baños, R. M., Quero, S., Alcañiz, M., \& Riva, G. (2007). Virtual reality exposure in the treatment of panic disorder and agoraphobia: A controlled study. Clinical Psychology and Pychotherapy, 14, 164-175.

Botella, C., Osma, J., García Palacios, A., Guillén, V., \& Baños, R. M. (2008). Treatment of complicated grief using virtual reality. A case report. Death Studies, 32, 674-692.

Botella, C., Pérez-Ara, M. A., Bretón-López, J., Quero, S., García Palacios, A. \& Baños, R. M. (2016). In vivo versus augmented reality exposure in the treatment of small animal phobia: a randomized controlled trial. Plos One 11(2): e0148237.

Botella, C., Quero, S., Lasso de la Vega, N., Baños, R. M., Guillén, V., García Palacios, A., \& Castilla, D. (2006). Clinical issues in the application of virtual reality to treatment of PTSD. In M. Roy (Ed.), Novel Approaches to the Diagnosis and Treatment of Posttraumatic Stress Disorder (pp. 183-195). Amsterdam: IOS Press.

Botella, J., \& Sanchez-Meca, J. (2015). Meta-análisis en Ciencias Sociales y de la Salud. Madrid, Spain: Síntesis

Botella, C., Serrano, B., Baños, R., \& García-Palacios, A. (2015). Virtual reality exposure-based therapy for the treatment of posttraumatic stress disorder. A review of its efficacy, the adequacy of the treatment protocol, and its acceptability. Neuropsychiatric Disease and Treatment, 11, 2533-45. doi: 10.2147/NDT.S89542. 
Bryant, R. A., Kenny, L., Joscelyne, A., Rawson, N., Maccallum, F., Cahill, C., ... \& Nickerson, A. (2014). Treating prolonged grief disorder: A randomized clinical trial. JAMA psychiatry, 71(12), 1332-1339.

Carta, M. G., Balestrieri, M., Murru. A., \& Carolina-Hardoy, M. C. (2009). Adjustment Disorder: epidemiology, diagnosis and treatment. Clinical Practice and Epidemioly in Mental Health, 5, 1-15.

Casey, P., \& Bailey, S. (2011). Adjustment disorders: the state of the art. World Psychiatry, 10, $11-18$.

Casey, P. (2009). Adjustment Disorder: epidemiology, diagnosis and treatment. CNS Drugs, 23, 927-938.

Casey, P., Jabbar, F., O'Leary, E., \& Doherty, A. M. (2015). Suicidal behaviours in adjustment disorder and depressive disorder. Journal of Affective Disorders, 174, 441-446.

Chakraborty, H., \& Gu, H. (2009). A mixed model approach for intent-to-treat analysis in longitudinal clinical trials with missing values (Report No. MR-0009-0903). Research Triangle Park, NC: RTI Press.

Comelius, L. R., Brouwer, S., de Boer, M. R. et al (2014). Development and validation of the Diagnostic Interview of Adjustment Disorder (DIAD). International Journal of Methods in Psychiatric Research, 23 (2), 192-207

Crunk, A.E., Burke, L.A. \& Robinson III, E.H.M. (2017). Complicated grief: An evolving theoretical landscape. Journal of Counseling \& Development, 95, 226-233.

Currier, J. M., Holland, J. M., \& Neimeyer, R. A. (2010). Do CBT-Based Interventions Alleviate Distress Following Bereavement? A Review of the Current Evidence. International Journal of Cognitive Therapy, 3, 77-93.

Cvetek, R. (2008). EMDR treatment of distressful experiences that fail to meet the criteria for PTSD. Journal of EMDR Practice and Research, 2 (1), 2-14.

Di Nardo, P. A., Brown, T. A., \& Barlow, D. H. (1994). Anxiety disorders interview schedule for DSM-IV: Lifetime Version (ADIS-IV), San Antonio, Texas.

Echeburúa, E., Corral, P., \& Fernández-Montalvo, J. (2000). Escala de inadaptación: propiedades psicométricas en contextos clínicos. Análisis y Modificación de Conducta, 26, 325-340.

Emmelkamp, P. M. G., Krijn, M., Hulsbosch, A. M., de Vries, S., Schuemie, M. J., \& van der Mast, C. A. P. G. (2002). Virtual reality treatment versus exposure in vivo: a comparative evaluation in acrophobia. Behaviour Research and Therapy, 40, 509-516. 
Foa, E. B., \& Kozak, M. J. (1986). Emotional processing of fear: Exposure to corrective information. Psychological Bulletin, 99, 20-35.

Freeman, D., Reeve, S., Robinson, A., Ehlers, A., Clarkm, D., Spanlang, B. \& Slater, M. (2017). Virtual reality in the assessment, understanding, and treatment of mental health disorders. Psychological Medicine. Doi: 10.1017/S003329171700040X

Goncalves, R., Pedrozo, A. L., Coutinho, E. S. F., Figueira, I., \& Ventura, P. (2012). Efficacy of Virtual Reality Exposure Therapy in the Treatment of PTSD: A Systematic Review. Plos One, 7, 12. e48469.

Gonzalez-Jaimes, E. I., \& Turnbull-Plaza, B. (2003). Selection of psychotherapeutic treatment for adjustment disorder with depressive mood due to acute myocardial infarction. Archives of Medical Research, 34, 298-304.

Gregg, L., \& Tarrier, N. (2007). Virtual reality in mental health: a review of the literature. Social Psychiatry and Psychiatric Epidemiology, 42, 343-354.

Gueorguieva, R., \& Krystal, J. H. (2004). Move Over ANOVA. Archives of General Psychiatry, 61(3), 310. doi:10.1001/archpsyc.61.3.310

Ho, S. M., Chu, K. W., \& Yiu, J. (2008). The relationship between explanatory style and posttraumatic growth after bereavement in a non-clinical sample. Death Studies, 32, 461478.

Hsiao, F. H., Lai, Y. M., Chen, Y. T., Yang, T. T., Liao, S. C., Ho, R. T. H., Ng, S. M., Chan, C. L., \& Jow, G. M. (2014). Efficacy of psychotherapy on diurnal cortisol patterns and suicidal ideation in adjustment disorder with depressed mood. General Hospital Psychiatry, 36, $214-$ 219.

Jacobson, N. S., \& Truax, P. (1991). Clinical significance: a statistical approach to defining meaningful change in psychotherapy research. Journal of Consulting and Clinical Psychology, 59, 12-19.

Kryzhanovskaya, L., \& Canterbury, R. (2001). Suicidal behavior in patients with adjustment disorders. Crisis. 22, 125-131.

Latham, A. E. y Prigerson, H. G. (2004). Suicide and Life-Threatening Behavior, 34 (4), 350362.

Lagerveld, S., Blonk, R., Brenninkmeijer, V., Wijngaards-de Meij, L. \& Schaufeli, W. (2012). Work-Focused Treatment of Common Mental Disorders and Return to Work: A Comparative Outcome Study. Journal of Occupational and Health Psychology, 17, 220-234. 
Li, J., Laursen, T. M., Precht, D. H., Olsen, J., \& Mortensen, P. B. (2005). Hospitalization for mental illness among parents after the death of a child. The New England Journal of Medicine, 352, 1190-6. doi: 10.1056/NEJMoa033160

Lindner, P., Miloff, A., Hamilton, W., Reuterskiöld, L., Andersson, G., Powers, M. \& Carlbring, P. (2017). Creating state of the art, next-generation Virtual Reality exposure therapies for anxiety disorders using consumer hardware platforms: Design considerations and future direction. Cognitive Behaviour Therapy, 46, 404-420. doi: $10.1080 / 16506073.2017 .1280843$

Lundorff, M., Holmgren, H., Zachariae, R., Farver-Vestergaard, I. \& M. O'Connor (2017). Prevalence of prolonged grief disorder in adult bereavement: A systematic review and metaanalysis. Journal of Affective Disorders, 212, 138-149.

Maercker, A., Brewin, C. R., Bryant, R. A., Cloitre, M., van, Ommeren., Jones, L. M., Humayan, A., Kagee, A., Llosa, A. E., Rousseau, C., Somasundaram, D. J., Souza, R., Suzuki, Y., Weissbecker, I., Wessely, S. C., First, M.B., \& Reed, G. M. (2013). Diagnosis and classification of disorders specifically associated with stress: proposals for ICD-11. World. Psychiatry. 12, 198-206.

McCann, R. A., Armstrong, C. M., Skopp, N. A., Edwards-Stewart, A., Smolenski, D. J., June, J. D., Metzger-Abamukong, M., \& Reger, G. M. (2014). Virtual reality exposure therapy for the treatment of anxiety disorders: An evaluation of research quality. Journal of Anxiety Disorders, 28, 625-631.

Meyerbröker, K., \& Emmelkamp, P. M. G. (2010). Virtual reality exposure therapy in anxiety disorders: a systematic review of process-and-outcome studies. Depression and Anxiety, 27, 933-944.

Molés, M., Quero, S., Nebot, S., Rachyla, I., \& López, M. T. (2014). Datos preliminares de validación del Inventario de Crecimiento Postraumático en población clínica española con trastornos relacionados con el estrés. Paper presented at II congreso nacional de psicología positiva. May, Oropesa (Spain).

Morina, N., Ijntema, H., Meyerbrökerm K \& Emmelkamp, P.M.G. (2015). Can virtual reality exposure therapy gains be generalized to real-life? A meta-analysis of studies applying behavioral assessments. Behaviour Research and Therapy, 74, 18-4.

Motraghi, T. E., Seim, R. W., Meyer, E. C., \& Morissette, S. B. (2014). Virtual reality exposure therapy for the treatment of posttraumatic stress disorder: A methodological review using CONSORT guidelines. Journal of Clinical Psychology, 70, 197-208. 
Neimeyer, R. A., \& Currier, J. M. (2009). Grief Therapy: Evidence of Efficacy and Emerging Directions. Current Directions in Psychological Science, 18, 352-356.

Neimeyer, R. A. (2000). Lessons of Loss. Florida: Keystone Heights.

Newman, M.G., Szkodny, L.E., Llera, S. J. \& Przeworski, A. (2011). A review of technologyassisted self-help and minimal contact therapies for anxiety and depression: Is human contact necessary for therapeutic efficacy?. Clinical Psychology Review, 31, 89-103.

Opriş, D., Pintea, S., García-Palacios, A., Botella, C., Szamosközi, S., \& David, D. (2012). Virtual reality exposure therapy in anxiety disorders: a quantitative meta-analysis. Depression and Anxiety, 29, 85-93.

Parsons, T. D., \& Rizzo, A. A. (2008). Affective outcomes of virtual reality exposure therapy for anxiety and specific phobias: A meta-analysis. Journal of Behavior Therapy and Experimental Psychiatry, 39, 250-261.

Pelkonen, M., Marttunen, M., Henriksson, M., \& Lönnqvist, J. (2007). Adolescent adjustment disorder: Precipitant stressors and distress symptoms of 89 outpatients. European Psychiatry, 22, 288-295.

Peterson, C., \& Seligman, M. E. (2004). Character strengths and virtues: A handbook and classification. Washington, DC: American Psychological Association.

Popper, K. (1995). La responsabilidad de vivir. Barcelona: Paidós.

Powers, M. B., \& Emmelkamp, P. M. (2008). Virtual reality exposure therapy for anxiety disorders: A meta-analysis. Journal of Anxiety Disorders, 22, 561- 569.

Prigerson, H. G., Bierhals, A. J., Kasl, S. V., Reynolds, C. F., Shear, M. K., Day, N., ...\& Jacobs, S. (1997). Traumatic grief as a risk factor for mental and physical morbidity. American Journal of Psychiatry, 154, 616-623.

Prigerson, H. G., Maciejewsku, P. K., Reynolds, C. F., Bierhals, A. J., Newsom, J. T., Fasiczka, A., Frank, E.,Doman, J., \& Miller, M. (1995). Inventory of Complicated Grief: A scale to measure maladaptive symptoms of loss. Psychiatry Research, 59, 65-79.

Prigerson, H., \& Jacobs, S. (2001). Traumatic grief as a distinct disorder: A rationale, consensus criteria, and a preliminary empirical test. In M. Stroebe, W. Stroebe, R. O. Hansson, \& H. Schut (Eds.), Handbook of bereavement research: Consequences, coping, and care. (pp. 613-645). Washington, DC: American Psychological Association Press.

Quero, S., Molés, M., Mor, S., Baños R., \& Botella, C. (2014). Spanish Validation of the Complicated Grief Inventory adapted for Adjustment Disorders. Paper presented at $A B C T^{\prime} S$ 48th Annual Convention. November, Philadelphia (USA). 
Quero, S., Molés, M., Pérez, M. A., Botella, C., \& Baños, R. M. (2012). An online emotional system to deliver homework assignments for treating adjustment disorders. In B. K. Wiederhold \& G. Riva (Eds.), Annual review of cybertherapy and telemedicine (pp. 273278). Interactive Media Institute, Amsterdam: IOS Press. ISBN: 978-1-61499-120-5.

Ready, D. J., Pollack, S., Rothbaum, B. O., \& Alarcon, R. D. (2006). Virtual reality exposure for veterans with posttraumatic stress disorder. Journal of Aggression, Maltreatment and Trauma, 12, 199-220.

Rothbaum, B. O., Hodges, L., Anderson, P. L., Price, L., \& Smith, S. (2002). Twelve-month follow-up of virtual reality and standard exposure therapies for the fear of flying. Journal of Consulting and Clinical Psychology, 70, 428-432.

Salim, A., Mackinnon, A., Christensen, H., \& Griffiths, K. (2008). Comparison of data analysis strategies for intent-to-treat analysis in pre-test-post-test designs with substantial dropout rates. Psychiatry Research, 160(3), 335-345. doi:10.1016/j.psychres.2007.08.005

Sanz, J., García-Vera, M-P., Espinosa, R., Fortún, M., \& Vázquez, C. (2005). Adaptación española del inventario para la depresión de Beck- II (BDI-II): Propiedades psicométricas en pacientes con trastornos psicológicos. Clínica y Salud, 16, 121-142.

Sanz, J., Navarro, M. E., \& Vázquez, C. (2003). Adaptación española del Inventario para la Depresión de Beck-II (BDI-II): Propiedades psicométricas en estudiantes universitarios. Análisis y Modificación de Conducta, 29, 239-288.

Sanz, J., Perdigón, A., \& Vázquez, C. (2003). Adaptación española del Inventario para la Depresión de Beck-II (BDI-II): 2. Propiedades psicométricas en población general. Clínica y Salud, 14, 249-280.

Shear, M.K. (2015). Complicated Grief. The New England Journal of Medicine, 372, 153-160.

Shear, M.K., Frank, E., Houck, P.R. \& Reynolds, C.F. (2005). Treatment of complicated grief: A randomized controlled trial. Journal of the American Medical Association, 293, 26012608 .

Shear, M.K., Wang, Y., Skritskaya, M., Duan, N., Mauro, C. \& Ghesquiere, A. (2014) Treatment of complicated grief in elderly persons: a randomized clinical trial. Journal of the American Medical Association, 71, 1287-1295.

Simon, N. M., Shear, K. M., Thompson, E. H., Zalta, A. K., Perlman, C., Reynolds, C. F. ... \& Silowash, R. (2007). The prevalence and correlates of psychiatric comorbidity in individuals with complicated grief. Comprehensive Psychiatry, 48(5), 395-399. 
So-Kum Tang, C. S. (2007). Posttraumatic growth of Southeast Asian survivors with physical injuries: Six months after the 2004 Southeast Asian earthquake-tsunami. Australasian Journal of Disaster and Trauma Studies, 1 ISSN: 1174-4707.

Srivastava, M., Talukdar, U., \& Lahan, V. (2011). Meditation for the management of adjustment disorder anxiety and depression. Complementary Therapies in Clinical Practices. $17,241-245$.

Steinhardt, M., \& Dolbier, C. (2008). Evaluation of a resilience intervention to enhance coping strategies and protective factors and decrease symptomatology. Journal of American College Health, 56, 445-453.

Strain, J. J. (1995). Adjustment disorders. In G. O. Gabbard (Ed.).Treatments of psychiatric Disorders, second ed. (pp. 1656-1665). Washington, DC: APA Press.

Stroebe, M. S., Schut, H. A. W., \& Stroebe, W. (2007). Health outcomes of bereavement. Lancet, 370, 1960-73. doi: 10.1016/S0140-6736(07)61816-9.

Tedeschi, R. G., \& Calhoun, L. G. (1996). The Posttraumatic Growth Inventory: measuring the positive legacy of trauma. Journal of Traumatic Stress, 9, 455-471.

Van der Klink, J. L., Blonk, R. W., Schene, A. H. \& van Dijk, F. J. (2003). Reducing long term sickness absence by an activating intervention in adjustment disorders. Occupational and Environmental Medicine, 60, 429-437.

World Health Organization (1992). International Classification of Diseases (ICD-10) $\left(10^{\text {th }}\right.$ Edition). Madrid: Meditor.

Yaseen, Y. A. (2017). Adjustment disorder: Prevalence, sociodemographic risk factors, and its subtypes in outpatient psychiatric clinic. Asian journal of psychiatry, 28, 8285 . 
Table 1. Participants' descriptive data about demographic variables, and diagnosis variables.

\begin{tabular}{|c|c|c|c|c|}
\hline & $\begin{array}{l}\text { EMMA } \\
(\mathrm{N}=18)\end{array}$ & $\begin{array}{c}\text { TRAD } \\
(\mathrm{N}=18)\end{array}$ & $\begin{array}{c}\text { WL } \\
(\mathrm{N}=18)\end{array}$ & $\begin{array}{c}\text { Total } \\
(\mathrm{N}=54)\end{array}$ \\
\hline \multicolumn{5}{|l|}{ Age } \\
\hline Range & $21-49$ & $18-42$ & $21-50$ & $18-50$ \\
\hline Mean (SD) & $34.67(8.63)$ & $30.06(7.83)$ & $34.67(8.35)$ & $33.13(8.41)$ \\
\hline \multicolumn{5}{|l|}{ Sex } \\
\hline Men & $4(22.2 \%)$ & $7(38.9 \%)$ & $3(16.7 \%)$ & $14(25.9 \%)$ \\
\hline Woman & $14(77.8 \%)$ & $11(61.1 \%)$ & $15(83.3 \%)$ & $40(74.1 \%)$ \\
\hline \multicolumn{5}{|l|}{ Marital status } \\
\hline Single & $7(38.9 \%)$ & $11(61.1 \%)$ & $8(44.4 \%)$ & $26(48.1 \%)$ \\
\hline Married/ partnered & $7(38.9 \%)$ & $7(38.9 \%)$ & $7(38.9 \%)$ & $21(38.9 \%)$ \\
\hline Divorced/separated/widower & $4(22.2 \%)$ & $0(0 \%)$ & $3(16.7 \%)$ & $7(13 \%)$ \\
\hline \multicolumn{5}{|l|}{ Level of studies } \\
\hline Elementary/High school & $3(16.7 \%)$ & $5(27.8 \%)$ & $6(33.3 \%)$ & $14(25.9 \%)$ \\
\hline University degree & $15(83.3 \%)$ & $13(72.2 \%)$ & $12(66.7 \%)$ & $40(74.1 \%)$ \\
\hline \multicolumn{5}{|l|}{ Diagnosis } \\
\hline \multicolumn{5}{|l|}{ AD subtype } \\
\hline With depressed mood & $2(11.1 \%)$ & $3(16.7 \%)$ & $2(11.1 \%)$ & $7(13 \%)$ \\
\hline With anxiety & $0(0 \%)$ & $1(5.6 \%)$ & $1(5.6 \%)$ & $2(3.7 \%)$ \\
\hline $\begin{array}{l}\text { With mixed anxiety and } \\
\text { depressed mood }\end{array}$ & $15(83.3 \%)$ & $12(66.7 \%)$ & $11(61.1 \%)$ & $38(70.4 \%)$ \\
\hline With disturbance of conduct & $0(0 \%)$ & $0(0 \%)$ & $1(5.6 \%)$ & $1(1.9 \%)$ \\
\hline $\mathbf{C G}$ & $1(5.6 \%)$ & $2(11.1 \%)$ & $3(16.7 \%)$ & $6(11.1 \%)$ \\
\hline \multicolumn{5}{|l|}{ Length of the problem } \\
\hline Range (months to years) & 3 to 20 & 3 to 17 & 5 to 10 & 3 to 20 \\
\hline Years mean (SD) & $4.37(5.29)$ & $3.11(4.02)$ & $3.51(3.38)$ & $3.66(4.26)$ \\
\hline \multicolumn{5}{|l|}{$\begin{array}{l}\text { Medication (anxiolytics } \\
\text { and/or antidepressants) }\end{array}$} \\
\hline Yes & $26.7 \%$ & $6.7 \%$ & $31.3 \%$ & $21.7 \%$ \\
\hline No & $73.3 \%$ & $93.3 \%$ & $68.8 \%$ & $78.3 \%$ \\
\hline
\end{tabular}


Table 2. Means, standard deviations and within-group comparisons effect sizes for primary and secondary outcome measures.

\begin{tabular}{|c|c|c|c|c|c|c|c|c|}
\hline & & $\begin{array}{c}\text { Pre } \\
(n=54)\end{array}$ & $\begin{array}{c}\text { Post } \\
(n=46)\end{array}$ & $\begin{array}{c}\text { 6-month FW } \\
(n=29)\end{array}$ & $\begin{array}{c}\text { 12-month FW } \\
(n=24)\end{array}$ & Pre vs. Post & Pre vs. 6-month FW & Pre vs. 12 month FW \\
\hline & & Mean (SD) & Mean (SD) & Mean (SD) & Mean (SD) & $d(95 \% \mathrm{CI})$ & $d(95 \% \mathrm{CI})$ & $d(95 \% \mathrm{CI})$ \\
\hline \multicolumn{9}{|l|}{$I S L / I C G$} \\
\hline & TRAD & $35.72(16.88)$ & $17.67(7.10)$ & $13.71(9.49)$ & $11.00(7.56)$ & $d=1.02(.55,1.50)$ & $d=1.25(.59,1.90)$ & $d=1.40(.59,2.21)$ \\
\hline & EMMA & $36.33(13.05)$ & $19.40(13.87)$ & $13.53(12.63)$ & $5.86(5.38)$ & $d=1.24(.48,2.00)$ & $d=1.67(.81,2.52)$ & $d=2.23(1.08,3.38)$ \\
\hline & WL & $31.25(15.93)$ & $28.53(14.53)$ & - & - & $d=.16(-.25, .58)$ & - & - \\
\hline \multicolumn{9}{|l|}{$B D I-I I$} \\
\hline & TRAD & $23.00(10.48)$ & $10.40(7.71)$ & $8.93(9.55)$ & $8.70(9.43)$ & $d=1.15(.40,1.89)$ & $d=1.28(.57,1.99)$ & $d=1.30(.58,2.02)$ \\
\hline & EMMA & $23.00(9.41)$ & $11.87(6.77)$ & $7.93(6.41)$ & $4.21(7.37)$ & $d=1.13(.40,1.86)$ & $d=1.53(.80,2.26)$ & $d=1.91(.93,2.89)$ \\
\hline & WL & $23.17(11.48)$ & $19.88(12.40)$ & - & - & $d=.16(-.09, .40)$ & - & - \\
\hline \multicolumn{9}{|l|}{ PTGI } \\
\hline & TRAD & $30.72(15.39)$ & $63.67(18.88)$ & $58.64(19.26)$ & $61.30(16.95)$ & $d=-2.05(-3.01,-.08)$ & $d=-1.73(-2.59,-.87)$ & $d=-1.57(-2.42,-.72)$ \\
\hline & EMMA & $29.50(18.61)$ & $61.13(24.10)$ & $56.00(21.71)$ & $63.29(19.19)$ & $d=-1.62(-2.33,-.92)$ & $d=-1.36(-2.02,-.70)$ & $d=-1.73(-2.53,-.94)$ \\
\hline & WL & $49.28(26.37)$ & $51.75(29.73)$ & - & - & $d=-.09(-.30, .12)$ & - & - \\
\hline \multicolumn{9}{|c|}{ Impairment } \\
\hline & TRAD & $5.78(1.00)$ & $3.21(1.81)$ & $2.36(1.34)$ & $2.00(1.41)$ & $d=2.45(1.44,3.46)$ & $d=3.27(1.92,4.62)$ & $d=.3 .61(2.05,5.18)$ \\
\hline & EMMA & $5.83(1.15)$ & $3.80(.86)$ & $2.00(1.73)$ & $1.43(1.28)$ & $d=1.69(.71,2.66)$ & $d=3.18(1.79,4.57)$ & $d=3.65(2.19,5.12)$ \\
\hline & WL & $5.83(1.38)$ & $5.33(2.44)$ & - & - & $d=.35(-.07, .77)$ & - & - \\
\hline \multicolumn{9}{|l|}{ Severity } \\
\hline & TRAD & $5.41(1.12)$ & $3.17(.75)$ & $2.33(1.53)$ & $1.33(.578)$ & $d=1.91(1.01,2.81)$ & $d=2.63(1.43,3.82)$ & $d=3.48(2.05,4.91)$ \\
\hline & EMMA & $5.59(.62)$ & $2.70(1.06)$ & $2.33(.58)$ & $1.25(.50)$ & $d=4.45(2.79,6.11)$ & $d=5.02(3.12,6.93)$ & $d=6.69(4.18,9.20)$ \\
\hline & WL & $4.82(1.51)$ & $5.29(1.50)$ & - & - & $d=-.30(-.55,-.04)$ & - & - \\
\hline
\end{tabular}

Note: Mean and standard deviations (SD) are represented. Pre= Pre-treatment. Post= Post-treatment. FW= Follow-up. $d=$ Cohen's $d$. CI= Confidence Interval. EMMA $=$

EMMA Intervention group; TRAD= Traditional Intervention group; WL = Waiting list control group; ISL= Inventory of Stress and Loss; ICG= Inventory of Complicated Grief; BDI-II= Beck Depression Inventory II; PTGI= Posttraumatic Growth Inventory; Impairment= Maladjustment Scale; Severity= Clinician Severity Scale. 
Table 3. Between-group comparisons at post-treatment and 6- and 12-month follow-Up.

\begin{tabular}{|c|c|c|c|c|c|c|c|}
\hline & & \multicolumn{2}{|c|}{ Post-treatment } & \multicolumn{2}{|c|}{ 6-month follow-up } & \multicolumn{2}{|c|}{ 12-month follow-up } \\
\hline & & $\begin{array}{l}\text { Mean } \\
\text { diff. }\end{array}$ & $d(95 \% \mathrm{CI})$ & $\begin{array}{l}\text { Mean } \\
\text { diff. }\end{array}$ & $d(95 \% \mathrm{CI})$ & $\begin{array}{l}\text { Mean } \\
\text { diff. }\end{array}$ & $d(95 \% \mathrm{CI})$ \\
\hline \multicolumn{8}{|l|}{$\overline{S L I / I C G}$} \\
\hline & TRAD vs. WL & $-10.38 *$ & $d=-.92(-1.68,-.17)$ & - & - & - & - \\
\hline & EMMA vs. WL & -9.65 & $d=-.63(-1.36, .11)$ & - & - & - & - \\
\hline & EMMA vs. TRAD & .74 & $d=.15(-.56, .87)$ & -.96 & $\begin{array}{l}d=-.02 \\
(-.74, .71)\end{array}$ & -5.27 & $\begin{array}{c}d=-.78 \\
(-1.62, .06)\end{array}$ \\
\hline \multicolumn{8}{|l|}{$B D I-I I$} \\
\hline & TRAD vs. WL & $-9.07 *$ & $d=-.89(-1.64,-.14)$ & - & - & - & - \\
\hline & EMMA vs. WL & $-7.53^{*}$ & $d=-.78(-1.52,-.04)$ & - & - & - & - \\
\hline & EMMA vs. TRAD & 1.53 & $d=.20(-.52, .91)$ & -1.19 & $\begin{array}{c}d=-.12 \\
(-.84, .61)\end{array}$ & -4.75 & $\begin{array}{c}d=-.58 \\
(-1.41, .24)\end{array}$ \\
\hline \multicolumn{8}{|l|}{ PTGI } \\
\hline & TRAD vs. WL & 12.43 & $d=.47(-.26,1.19)$ & - & - & - & - \\
\hline & EMMA vs. WL & 9.13 & $d=.34(-.37,1.06)$ & - & - & - & - \\
\hline & EMMA vs. TRAD & -3.30 & $d=-.11(-.82, .61)$ & -1.55 & $\begin{array}{c}d=-.12 \\
(-.85, .60)\end{array}$ & 2.24 & $\begin{array}{c}d=.11 \\
(-.70, .92)\end{array}$ \\
\hline \multicolumn{8}{|l|}{ Impairment } \\
\hline & TRAD vs. WL & $-2.06 * * *$ & $d=-.96(-1.72,-.20)$ & - & - & - & - \\
\hline & EMMA vs. WL & $-1.43 * *$ & $d=-.81(-1.56,-.07)$ & - & - & - & - \\
\hline & EMMA vs. TRAD & .62 & $d=.41(-.32,1.13)$ & -.30 & $\begin{array}{l}d=-.23 \\
(-.96, .51)\end{array}$ & .51 & $\begin{array}{c}d=-.41 \\
(-1.23, .41)\end{array}$ \\
\hline \multicolumn{8}{|l|}{ Severity } \\
\hline & TRAD vs. WL & $-1.64 * *$ & $d=-1.74(-2.58,-.90)$ & - & - & - & - \\
\hline & EMMA vs. WL & $-2.25 * * *$ & $d=-1.94(-2.81,-1.07)$ & - & - & - & - \\
\hline & EMMA vs. TRAD & -.62 & $d=-.50(-1.22, .23)$ & .072 & $\begin{array}{c}d=.00 \\
(-.73, .73)\end{array}$ & .53 & $\begin{array}{l}d=-.14 \\
(-.96, .67)\end{array}$ \\
\hline
\end{tabular}

Note: $d=$ Cohen's $d$. Values between () represents the 95\% Confidence Interval. Mean diff. $=$ Mean differences. $* \mathrm{p}<.05, * *$ $\mathrm{p}<.01, * * * \mathrm{p}<.001$. EMMA = EMMA Intervention group; TRAD= Traditional Intervention group; WL $=\mathrm{Waiting}$ list control group; ISL= Inventory of Stress and Loss; ICG= Inventory of Complicated Grief; BDI-II= Beck Depression Inventory II; PTGI= Posttraumatic Growth Inventory; Impairment= Maladjustment Scale; Severity= Clinician Severity Scale. 
Table 4. Clinically Meaningful Improvement on the ISL/ICG and BDI at Post-treatment, 6 and 12 month follow up.

\begin{tabular}{|c|c|c|c|c|c|c|}
\hline \multirow[b]{2}{*}{ Measures } & \multicolumn{2}{|c|}{ Post-treatment } & \multicolumn{2}{|c|}{ 6-month FW } & \multicolumn{2}{|c|}{ 12-month FW } \\
\hline & EMMA & TRAD & EMMA & TRAD & EMMA & TRAD \\
\hline \multicolumn{7}{|l|}{ ISL/ICG } \\
\hline Recovered & $10(71.4 \%)$ & $10(66.7 \%)$ & $12(85.7 \%)$ & $11(84.6 \%)$ & $13(100 \%)$ & $7(70 \%)$ \\
\hline Improved & $0(0 \%)$ & $0(0 \%)$ & $0(0 \%)$ & $0(0 \%)$ & $0(0 \%)$ & $0(0 \%)$ \\
\hline No change & $4(28.6 \%)$ & $5(33.3 \%)$ & $2(14.3 \%)$ & $2(15.4 \%)$ & $0(0 \%)$ & $3(30 \%)$ \\
\hline Impaired & $0(0 \%)$ & $0(0 \%)$ & $0(0 \%)$ & $0(0 \%)$ & $0(0 \%)$ & $0(0 \%)$ \\
\hline \multicolumn{7}{|l|}{ BDI-II } \\
\hline Recovered & $4(26.7 \%)$ & $6(40 \%)$ & $8(53.3 \%)$ & $6(42.9 \%)$ & $10(71.4 \%)$ & $3(30 \%)$ \\
\hline Improved & $1(6.7 \%)$ & $0(0 \%)$ & $1(6.7 \%)$ & $1(7.1 \%)$ & $0(0 \%)$ & $0(0 \%)$ \\
\hline No change & $10(66.7 \%)$ & $9(60 \%)$ & $6(40 \%)$ & $7(50 \%)$ & $4(28.6 \%)$ & $7(70 \%)$ \\
\hline Impaired & $0(0 \%)$ & $0(0 \%)$ & $0(0 \%)$ & $0(0 \%)$ & $0(0 \%)$ & $0(0 \%)$ \\
\hline
\end{tabular}

